\title{
Preparação do enfermeiro para o atendimento à múltiplas vítimas no resgate aéreo
}

RESUMO |Objetivo: Caracterizar a atuação do enfermeiro de bordo, com ênfase no atendimento à múltiplas vítimas. Metodologia: Estudo exploratório e qualitativo. Os dados foram obtidos por meio de questionários semiestruturados, aplicados nosenfermeiros debordo em Belo Horizonte/MG. Utilizada a técnica metodológica de Bola de Neve (Snowball Sampling) e analisesob o conteúdo de Laurence Bardin. Resultado: Dos enfermeiros atuantes no resgate aéreo, que aceitaram participar, a maioria são do sexo masculino, possuem a renda familiar média de nove salários mínimos, mais de um vínculo empregatício, experiência média de seis anos. A partir da análise dos dados, as categorias elencadas foram: Atendimento Sistematizado e Capacitação Aeromédica. Conclusão: É preciso reconhecer o papel do enfermeiro no atendimento as vítimas no resgate aeromédico. Vale ressaltar a importância da capacitação para alcançar a excelência, no ambiente hostil confinado.

Palavras-chaves: Enfermeiros; Incidentes Com Feridos Em Massa; Trabalho De Resgate; Resgate Aéreo; Gestão Do Conhecimento.

ABSTRACT | Objective: To characterize the role of the nurse on board, with an emphasis on attending to multiple wounds. Methodology: Exploratory and qualitative study. Data were obtained through semi-structured, scientific questionnaires on board nurses in Belo Horizonte / MG. The Snowball methodological technique was used and analyzed under the content of Laurence Bardin. Result: Of the nurses working in the air rescue, who agreed to participate: the majority are male, have an average family income of nine relative, more than one job, average experience of six years. From the analysis of the data, according to the categories listed were: Systematized Service and Aeromedical Training. Conclusion: It is necessary to recognize the role of the nurse in the care aswounds in the aeromedical rescue. It is worth emphasizing the importance of training to achieve excellence in a confined hostile environment.

Keywords: Nurses; Incidents With Mass Injuries; Rescue Work; Aerial Rescue; Knowledge Management.

RESUMEN | Objetivo: Caracterizar el rol de la enfermera a bordo, con énfasis en la atención de múltiples heridas. Metodología: Estudio exploratorio y cualitativo. Los datos se obtuvieron a través de cuestionarios científicos semiestructurados a bordo de enfermeras en Belo Horizonte / MG. Se utilizó y analizó la técnica metodológica Snowball bajo el contenido de Laurence Bardin. Resultado: De los enfermeros que laboran en el rescate aéreo, que aceptaron participar: la mayoría son hombres, tienen un ingreso familiar promedio de nueve parientes, más de un trabajo, experiencia promedio de seis años. Del análisis de los datos, según las categorías enumeradas fueron: Servicio Sistematizado y Formación Aeromédica. Conclusión: Es necesario reconocer el papel del enfermero en el cuidado comoheridas en el rescate aeromédico. Vale la pena enfatizar la importancia de la capacitación para lograr la excelencia en un ambiente hostil confinado.

Palabras claves: Enfermeros; Incidentes Con Lesiones Masivas; Labores De Rescate; Rescate Aéreo; Gestión Del Conocimiento.

\section{Bruno Gonçalves da Silva}

Enfermeiro (UNIFENAS), Mestre em Administração de Empresas (Centro Universitário UNA), Doutorando em Sistemas de Informações e Gestão do Conhecimento (Universidade FUMEC). Professor Assistente FCMMG e Enfermeiro de Bordo Unimed Aeromédica.

ORCID: 0000-0001-5173-0036

\section{Leticia Lorrany Viana}

Enfermeira (FCMMG).

ORCID: 0000-0002-7073-8372

\section{Samantha de Souza Faustino}

Enfermeira (FCMMG).

ORCID: 0000-0002-0797-6466

\section{Carla de Paula Silveira}

Enfermeira. Professora Assistente FCMMG. Mestre em Educação em Diabetes (Faculdade Santa Casa).Professora Assistente FCMMG.

ORCID: 0000-0003-3092-5218

\section{Vânia Paula de Carvalho}

Enfermeira, Enfermeira RT da Unimed Aeromédica. Mestre em Promoção da Saúde e Prevenção da Violência (UFMG).

ORCID: 0000-0002-9336-3606

\section{Armando Sérgio de Aguiar Filho}

Comunicador Soaical, Professor Titular na Universidade FUMEC e Faculdade Promove. Doutor em Gestão da Informação e do Conhecimento (UFMG).

ORCID: 0000-0001-5542-7165

Recebido em: 26/04/2021

Aprovado em: 01/06/2021
INTRODUÇÃO

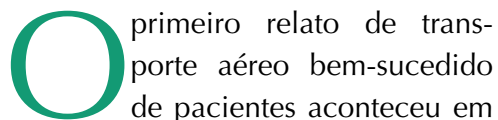
1870, no cerco de Paris por alemães, em balões de ar quente. Houve uma série de eventos na linha histórica do transporte aéreo de pacientes críticos, mundialmente. Neste contexto, considerando os agravantes em nosso país, tais como: horários de pico no trânsito, locais de difícil acesso, escassez de ambulância, distância de unidades hospitalares e dentre outros, instituiu-se o resgate aeromédico composto por médico, enfermeiro e piloto ${ }^{(1)}$.

Em Minas Gerais (MG), a equipe interdisciplinar atuante no Batalhão de Operações Aéreas do Corpo de Bombeiros Militar (BOA) é composta por bombeiros militares, com as mais diversas 
funções. Mantém há anos, uma parceria com os profissionais do Serviço de Atendimento Móvel de Urgência (SAMU 192), sendo composta por médicos e enfermeiros, no atendimento às vítimas ${ }^{(2)}$.

No Estado, o arcabouço para a criação de um plano de atendimento de serviços de urgência e emergência a desastres e catástrofes surgiu devido à necessidade de um programa preventivo de atendimento a dois grandes eventos: Copa das Confederações em 2013, Copa do Mundo de Futebol em 2014 e Olimpíadas em $2016^{(3)}$.

$\mathrm{O}$ atendimento pré-hospitalar (APH) ocorre no cotidiano de forma inesperada e as ocorrências podem ser constituídas por casos individuais ou incidentes com múltiplas vítimas (IMV) ${ }^{(4)}$. Em um IMV, o atendimento prestado tem como objetivo atender o maior número de vítimas, no menor tempo possível. Para isso, é necessário realizar a triagem através do método Simple Triage and Rapid Treatment $(\mathrm{START})^{(5)}$.

A triagem pelo método START é utilizada mundialmente em situações de ocorrências com múltiplas vítimas, busca promover o tratamento inicial, com estabilização e transporte para a referência através de ações controladas por meio de regulação e comunicação efetiva. No Brasil, o Ministério da Saúde recomenda a realização de triagem em casos de eventos com cinco vítimas ou mais ${ }^{(5,6)}$.

O Conselho Federal de Enfermagem (COFEN), por meio da Lei $\mathrm{n}^{\circ} 5.905$ de 12 de julho de 1973, através da resolução $n^{\circ} 379 / 2011$, predispõe a presença da enfermagem no $\mathrm{APH}$ frente o suporte básico e avançado de vida, prestados por profissionais devidamente habilitados, assegurados pela lei do exercício profissional $n^{\circ} 7.498 / 86^{(7)}$. Considerando uma linha histórica do enfermeiro de bordo: A Resolução de $n^{\circ}$ 551, de 12 de julho de 2017, normatizou a atuação do enfermeiro no APH móvel e inter-hospitalar em veículo aéreo ${ }^{(8)}$. A Resolução COFEN $n^{\circ}$ 660/2021, de 03 de março de 2021, altera a Resolução COFEN n 656, de 17 de dezembro de 2020, que normatizou a atuação do enfermeiro na assistência direta e no gerenciamento do $\mathrm{APH}$ e Inter-hospitalar em veículo aéreo ${ }^{(9,10)}$.

O enfermeiro, frente a um IMV, tem a atribuição de avaliar a gravidade das lesões e aplicar manobras para a manutenção da vida, favorecendoo aumento da sobrevida. Assim como, é capacitado para fornecer um atendimento de

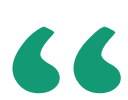

\section{Trata-se de uma pesquisa descritiva exploratória, com abordagem qualitativa, por intermédio de questionário semiestruturado sobre a atuação do enfermeiro de bordono atendimento a múltiplas vítimas em um serviço de resgate aéreo.}

qualidade ao maior número de vítimas possíveis $^{(8,11)}$.

Diante do exposto, este estudo teve como objetivo descrever aspectos da atuação do enfermeiro de bordo, com ênfase no atendimento à múltiplas vítimas.

\section{MÉTODO}

Tipo de estudo

Trata-se de uma pesquisa descritiva exploratória, com abordagem qualitativa, por intermédio de questionário semiestruturado sobre a atuação do enfermeiro de bordono atendimento a múltiplas vítimas em um serviço de resgate aéreo.

A pesquisa exploratória visa proporcionar um aprofundamento no problema proposto a fim de possibilitar seu entendimento de forma clara. A abordagem qualitativa adota uma avaliação subjetiva e empírica, possibilitando que o pesquisador, por meio da análise das entrevistas, interaja com o entrevistado. Assim, o pesquisador pretende compreender as aspirações, valores, crenças e atitudes dos entrevistados. No entanto, tais aspectos não podem ser quantificados ${ }^{(12)}$.

Os entrevistados na pesquisa são enfermeiros atuantes no resgate aeromédico, da rede pública, na região metropolitana de Belo Horizonte/MG. O serviço conta comsete profissionais, dos quais quatro foram entrevistados e com esse número foi possível obter a saturação dos dados. Os sujeitos receberam a letra inicial ' $E$ ' e a numeração em ordem crescente das entrevistas, preservando assim o anonimato de suas identidades.

O presente estudo seguiu orientações expressas na Resolução MS 466/12 do Conselho Nacional de Saúde que apresenta as normas éticas para a realização de pesquisas com seres humanos ${ }^{(13)}$. Foi aprovado no Comitê de Ética e Pesquisa (CEP) da Faculdade Ciências Médicas de Minas Gerais (FCM-MG), sob o CAAE: 15734719.9.0000.5134. A coleta de dados foi realizada através de um questionário semiestruturado após apreciação do CEP. Foi realizado teste piloto para 
verificar a consistência do questionário elaborado, antecedendo a sua aplicação. Os convidados a participar da pesquisa foram incluídos somente após assinatura do Termo de Consentimento Livre e Esclarecido (TCLE).

Após a coleta dos dados, as informações foram tabuladas e analisadas segundo a técnica de análise de conteúdo desenvolvida por Laurence Bardin. As etapas da técnica de análise das comunicações visaram obter, por procedimentos sistemáticos e objetivos de descrição do conteúdo das mensagens, indicadores (quantitativos ou não) que permitam a inferência de conhecimentos relativos às condições de produção/recepção destas mensagens. A análise foi realizada em três fases, descritas em: unidade de registro, unidade de contexto e unidade de significado, conforme apresentado na Figura $1^{(14)}$.

\section{RESULTADOS E DISCUSSÃO}

Dos seteenfermeiros atuantes no resgate aeromédico, quatro enfermeiros aceitaram participar da pesquisa
$(4 / 57,1 \%)$. Sendo que, durante a coleta de dados, um dos profissionaisestava de férias, outro não se sentiu à vontade para participar e o último estava fora do Estado (3/42,9\% ou $14,3 \%$ de cada um dos três).

Dentre os profissionais entrevistados, a maioria são do sexo masculino, possuem a renda familiar média de nove salários mínimos, mais de um vínculo empregatício, graduados há mais de quinze anos e experiência média de seis anos no atendimento a resgate aéreo (3/75\%). Todos os entrevistados possuem pós-graduação e metade possuem outros níveis de escolaridade como mestrado, doutorado e/ou MBA. Em relação ao estado civil, metade dos profissionais são casados. A partir da leitura sistemática das entrevistas, emergiram duas categorias: Atendimento Sistematizado e Capacitação Aeromédica.

\section{Atendimento Sistematizado}

O enfermeiro de bordo acumula as funções gerenciais e assistenciais. Cabe ao profissional realizar o planejamento durante todas as etapas do atendimento

\section{Figura 1- Desenvolvimento da análise BARDIN (1977)}

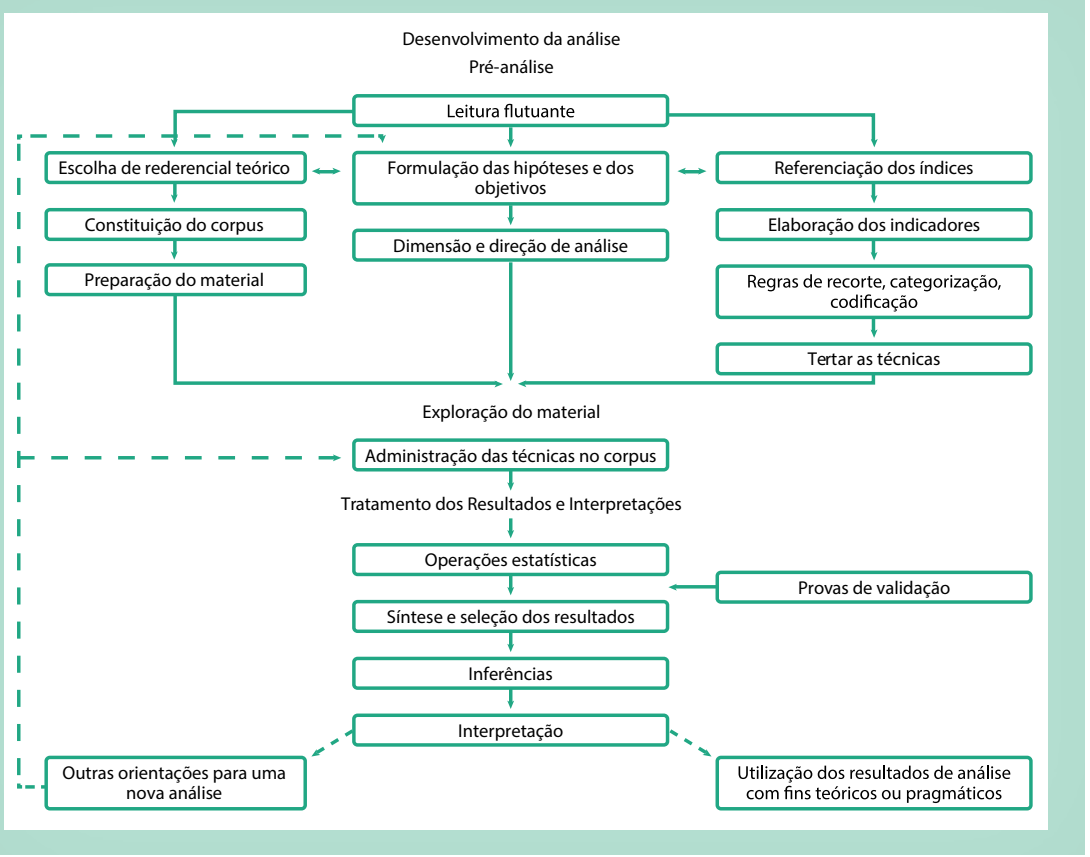

Fonte:Elaborado pelos autores, 2020 (pré, trans e pós) de forma que a Sistematização da Assistência de Enfermagem (SAE) esteja presente durante todo processo do transporte aeromédico ${ }^{(15)}$.

Oliveira, et al. ${ }^{(16)}$ em seu estudo, corroboram com Scuissiato, et al. ${ }^{(15)}$ descrevendo a SAE como uma metodologia que organiza todo o processo assistencial da enfermagem baseada em um delineamento orientado através de bases teóricas e filosóficas.

Diante das respostas obtidas, podemos evidenciar que os entrevistados entendiam o papel gerencial do enfermeiro de voo, frente ao atendimento prestado no transporte aéreo, através das seguintes frases:

"Primeiro, o enfermeiro tem um papel fundamental na questão de gerenciamento do atendimento a múltiplas vítimas [...] ele tem que ter o conhecimento de quais materiais estão disponíveis para o atendimento, dos hospitais[...] que recebem esses pacientes e a questão mesmo de como ele vai triar esses pacientes." E2

"Bom, o enfermeiro é muito importante no papel de gerir os recursos que a gente tem, recursos humanos, recursos técnicos, recursos materiais, prevê e provê esse material [...]" E3

O gerenciamento prestado pelo enfermeiro em situações de desastres é uma importante ferramenta para minimizar a ocorrência de eventos adversos que possam acometer a equipe e as vítimas presentes no local. Através do conhecimento, o enfermeiro é capaz de gerenciar e dimensionar problemas, além de promover a segurança e uma resposta efetiva ${ }^{(17)}$.

\section{Capacitação Aeromédica}

Segundo Salvador et al. ${ }^{(18)}$, o IMV conta com situações específicas. Nesse contexto, a capacitação e o treinamento dos profissionais são primordiais, desde o início da sua formação acadêmica. 
A capacitação qualificada pode fazer com que os enfermeiros se sintam aptos para atuarem, otimizando a segurança, a resolubilidade e minimizando a incidência de possíveis eventos adversos, durante o atendimento ao paciente criticamente enfermo.

Os relatos seguintes retratam a importância do conhecimento teórico e prático do enfermeiro no serviço aeromédicodurante o atendimento a vítima.

"[...] é um serviço muito limitado, um serviço que é novo no sistema, exige do profissional conhecimentos aeroespaciais, experiência profissional em urgência e emergência ou terapia intensiva $[\ldots]^{\prime \prime} \mathrm{E} 2$

"[...] vai exigir de você conhecimento, vai exigir de você determinadas habilidades porque ele é um ambiente hostil [...]" E4

Para tanto,os profissionais devem ser preparados adequadamente,capacitados e treinados para prestaremo melhor atendimentopossível. O que irá assegurar o desenvolvimento de técnicas de salvamento relevantes, organizadas, eficazes, seguras e mitigando possíveis erros durante a atuação em situações de urgência e emergência (19).

Há várias particularidades enfrentadas pelos profissionais de saúde do serviço aéreo, tais como: a limitação do espaço na aeronave, a presença de ruídos, as acelerações, as desaceleraçõesas vibrações, dentre outras. Todas pontuações, podem gerar alterações fisiológicas em graus diferentes nos tripulantes ${ }^{(20)}$.

No que se refere às limitações e as especificidades enfrentadas pelos profissionais entrevistados, podemos explicitar os seguintes trechos:

"[...] tem a questão da limitação meteorológica, limitação de tempo [...] e tem também a questão da limitação de espaço, de condições de realizar a intervenção do paciente durante o transporte [...]" E1

"O transporte aéreo tem diversas especificidades [...] o espaço físi$\mathrm{CO}$, as condições referentes a alterações de pressão, de vibração, de temperatura [...] são diferentes em terra.Então no aéreo a gente tem que ter os equipamentos próprios [...]" E3

As alterações fisiológicasocorrem, tanto nos pacientes quanto na equipe, com o aumento da altitude da aeronave; como estresse de voo (vibração, umidade, ruídos, temperatura) e a aerodilatação, decorrente das constantes alterações de pressurizaçãodentro da aeronave $\mathrm{i}^{(20,21,22,23)}$. Sendo assim os autores, Scuissiato et al. ${ }^{(15)}$ reforçam a importância,para oprofissional atuante no transporte aeromédico, de dominar os conhecimentos inerentes de sua profissão, assim como, a fisiologia de voo.

Em seu estudo, Bonuzzi, et al. ${ }^{(21)}$ abordam a Portaria GM 2.048 de 5 de novembro de 2002, que institui a qualificação do profissional do transporte aeromédico e criação da especialidade de Enfermagem Aeroespacial, deferida pelo COFEN através Resolução 260/2001. Os autores apontam que o conhecimento específico apresenta benefícios na prática do enfermeiro de bordo no que concerne ao atendimento à vítima.

Os entrevistados, ao serem questionados sobre os treinamentos realizados no resgate aéreo, forneceram os seguintes relatos:

"[...] são realizados treinamentos de habilitação, o curso chama ASA, e qualifica médicos e enfermeiros para o atendimento aeromédico, tanto na parte da aeronáutica quanto na parte de atendimento [...]" E1

"Hoje nós temos o curso suporte avançado aeromédico aqui no Batalhão de Operações Aéreas do corpo de Bombeiros e vinculado ao SAMU de Belo horizonte, e existe a segurança de voo, existe o curso da tripulação para mexer no aeromédico e existe o curso de Atendimento à vítima e no serviço aeromédico." E2

Lamet al. ${ }^{(24)}$ afirmam que o planejamento, o treinamento e a preparação prévia dos profissionais no IMVmaximizama confiança, o conhecimento e as habilidades clínicas. Somados, a habilidade profissional, em detrimento das particularidades do serviço aeromédico, da necessidade de um tempo-resposta reduzido no atendimento às vítimas de trauma, da agilidade do modal, da especificidade do ambiente e do tratamento definitivo em um menor período ${ }^{(25)}$.

É acionado o Plano Integrado de Atendimento a Desastres com Múltiplas Vítimas, quando se trata de atendimento a IMV. Esse plano promove uma resposta integrada entre o serviço de saúde e segurança pública, com intuito de melhorar a comunicação entre os setores envolvidos o gerenciamento dos pacientes em redes hospitalares ${ }^{(3)}$.

Segundo relato dos entrevistados, os simulados são necessários, em conjunto com todos os setores envolvidos, a fim de melhorar o atendimento prestado às múltiplas vítimas. Tal fato fica explicitado no seguinte trecho:

"Acho que umas das principais fragilidades é a integração de diversos setores porque o atendimento com múltiplas vítimas nunca atende somente um órgão,SAMU, bombeiro e polícia, serviço de trânsito, defesa civil, todo mundo junto [...]" E3

Lima et al. corroboram, afirmando que a educação através da simulação realística,auxilia no desenvolvimento de habilidades técnicas, de comunicação e de trabalho em equipe. Ainda, a utilização dessa ferramenta, em ambientes controlados, contextualizados, otimiza o atendimento multiprofissional no cenário pré-hospitalar, em especial nos IMV. ${ }^{(19)}$ 
CONCLUSÃO

O estudo possibilitou conhecer algumas peculiaridades do enfermeiro no resgate aeromédico, a importância dasua capacitação profissional, da sistematização da assistência, além da percepçãoda otimizaçãoda qualidade assistencial.

As ações do enfermeiro de bordo abrangem diversos aspectos, gerenciais, tais como: planejar escalas, realizar provisão e previsão de materiais e equipamentos, organizar o atendimento aos IMV, dentre outros. Assim como, as atividades assistenciais sistematizadas, proporcionando ao paciente uma assistência segura e maior chancede sobrevida.

É notório que o resgate aéreo traz desafios a todos os profissionais inseridos no atendimento às vítimas e questões associadas ao ambiente de trabalho. Assim como, é um meio de transporte eficiente e seguro, capaz de proporcionar assistência integral, eficaz e rápida a vítimas.

Diante do exposto, esse estudo traz uma reflexão acerca da importância da atuação do enfermeiro no atendimento à múltiplas vítimas no resgate aeromédico, por permitir vislumbrar a importância das competências, das habilidades essenciais para a atuação em uma área de alta performance e que exige acurácia no conhecimento científico, alinhado ao ambiente de trabalho.

Assim, ressalta-se a importância da realização de novosestudos em todo território nacional. Que abarquem a atuação do enfermeiro de bordo, sua relevância social e o investimento em políticas que possam proporcionar ao profissional uma maior visibilidade.

\section{Referências}

1. Cardoso, R. G., Francischini, C. F., Ribera, J. M., Vanzetto, R. I. C. A. R. D. 0., \& Fraga, G. P. (2014). Resgate aeromédico a traumatizados: experiência na região metropolitana de Campinas, Brasil. Rev Col Bras Cir, 41(4), 236-44. 2. Secretaria do Estado de Saúde de Minas Gerais. Parceria entre SES-MG e Corpo de Bombeiros que salva vidas.08/03/2019. Disponível em: https://www. saude.mg.gov.br/component/gmg/story/10946-parceria-entre-ses-mg-e-corpo-de-bombeiros-que-salva-vidas

3. Gerais, C. N. E. D. M. Organização da Atenção à Saúde no Âmbito Pré-hospitalar e Hospitalar para Enfrentamento de Situações de Múltiplas Vítimas, Desastres e Catástrofes no Estado de Minas Gerais: Legislação, Estrutura Física, e capacitação de profissionais. Brasília (DF); 2013.

4. Damasceno, M. C. D. T., \& Ribera, J. M. (2012). Desastres e incidentes com múltiplas vítimas: plano de atendimento: preparação hospitalar. In Desastres e incidentes com múltiplas vítimas: plano de atendimento: preparação hospitalar (pp. 39-39).

5. Simões, R. L., Duarte Neto, C., Maciel, G. S. B., Furtado, T. P., \& Paulo, D. N. S. (2012). Atendimento pré-hospitalar à múltiplas vítimas com trauma simulado. Rev. Col. Bras. Cir, 230-237.

6. Brasil. Ministério da Saúde (2016). Secretaria de Atenção à Saúde. Protocolos de intervenção para o SAMU 192- Serviço de Atendimento Móvel de Urgência. Brasília: Ministério da Saúde; $2^{\mathrm{a}}$ edição.

7. Brasil. Conselho Federal de Enfermagem (COFEN). Resolução $n^{0} .379 / 2011$ : Dispõe da presença do Enfermeiro no Atendimento Pré-Hospitalar e Inter-Hospitalar. Brasília (DF); 2011

8. Brasil. Conselho Federal de Enfermagem (COFEN). Resolução no.551/2017: Enfermeiro que atua no serviço de atendimento pré-hospitalar ou inter-hospitalar através de aeronaves. Brasília (DF); 2017.

9. Brasil. Conselho Federal de Enfermagem (COFEN). Resolução nº.660/2021:Altera a resolução COFEN 656, de 17 de dezembro de 2020, que normatiza a atuação do enfermeiro na assistência direta e no gerenciamento do atendimento pré-hospitalar móvel e inter-hospitalar em veículo aéreo. Brasília (DF) 2021.

10. Brasil. Conselho Federal de Enfermagem (COFEN). Resolução $n^{\circ} \cdot 656 / 2020$. Brasília:Normatiza a atuação do enfermeiro na assistência direta e no gerenciamento do atendimento pré-hospitalar móvel e inter-hospitalar em veículo aéreo. (DF); 2020.

11. de Campos, A. L. (2015). Atendimento de Emergência Realizado por Profissionais de Enfermagem, Médico, Bombeiros e Demais Profissionais Treinados a Vítimas de Acidentes e Catástrofes. Revista de Medicina e Saúde de Brasília, $4(1)$.

12. Gerhardt, T. E., \& Silveira, D. T. (2009). Métodos de pesquisa. Plageder. 13. Brasil, M. Conselho Nacional de Saúde, Resolução $n^{\circ} 466$, de 12 de dezembro de 2012. Diretrizes e normas regulamentadoras de pesquisas envol- vendo seres humanos. Diário Oficial da União República Federativa do Brasil, 150(112).

14. Bardin, L. (2011). Análise de conteúdo 4ed. Lisboa: Edições, 70, 1977.

15. Scuissiato, D. R., Boffi, L. V., Rocha, R. D. R. D., Montezeli, J. H., Bordin, M. T., \& Peres, A. M. (2012). Compreensão de enfermeiros de bordo sobre seu papel na equipe multiprofissional de transporte aeromédico. Revista Brasileira de Enfermagem, 65(4), 614-620.

16. Oliveira, M. R. D., Almeida, P. C. D., Moreira, T. M. M., \& Torres, R. A. M. (2019). Sistematização da assistência de enfermagem: percepção e conhecimento da enfermagem Brasileira. Revista Brasileira de Enfermagem, 72(6), 1547-1553.

17. Martiniano, E. C., do Nascimento, A. M. V., Campos, J. R. E., Campos, J. B. R. Barros, A. B., \& Luz, D. C. R. P. (2020). Cuidados de enfermagem ao paciente politraumatizado: revisão integrativa. Nursing (São Paulo), 23(270), 4861-4872. 18. Salvador, P. T. C. D. O., Dantas, R. A. N., Dantas, D. V., \& Torres, G. D. V. (2012). A formação acadêmica de enfermagem e os incidentes com múltiplas vítimas: revisão integrativa. Revista da Escola de Enfermagem da USP, 46(3), 742-751. 19. Lima, D. S., de-Vasconcelos, I. F., Queiroz, E. F., Cunha, T. A., dos-Santos, V. S., Arruda, F. A. E. L., \& Freitas, J. G. (2019). Simulação de incidente com múltiplas vítimas: treinando profissionais e ensinando universitários. Revista do Colégio Brasileiro de Cirurgiões, 46(3).

20. Lima, E. W. M., Mota, M. H. L. V., Rocha, F. C. V. V., Madeira, M. Z. A., \& Oliveira, A. D. S. (2015). Satisfaction of nurses in service mobile service emergency air/Satisfação do enfermeiro no serviço de atendimento móvel de urgência aéreo/Satisfacción de enfermeras en servicio de servicio móvil de aire de emergência. Revista de Enfermagem da UFPI, 4(4), 49-55.

21. Bonuzzi, K. L., Muniz, C. C. S. D. S., Santos, O. P. D., Moraes Filho, I. M. D., Lopes, V. C., \& Silva, R. M. D. (2016). Atuação do enfermeiro no atendimento pré hospitalar aéreo a pacientes politraumatizados-Revisão de literatura. Revista de Divulgação Científica Sena Aires, 5(2), 171-177.

22. JM, F. (2018). Principles of air transport physiology for JMATT clinicians. Retrieved Jan, 27.

23. Pena Dias, C., Lopes Ferreira, F., \& Paula de Carvalho, V. (2017). The Importance of Teamwork in Patient Air Transportation. Journal of Nursing UFPE/ Revista de Enfermagem UFPE, 11(6).

24. Lam, N. N., Huong, H. T. X., \& Tuan, C. A. (2018). Preparation for major burns incidents: evaluation of continuing medical education training courses for professionals. Annals of burns and fire disasters, 31(4), 322.

25. Schweitzer, G., Nascimento, E. R. P. D., Nascimento, K. C. D., Moreira, A. R., Amante, L. N., \& Malfussi, L. B. H. D. (2017). Intervenciones de emergencia realizadas en las víctimas de trauma de un servicio aéreo médico. Revista Brasileira de Enfermagem, 70(1), 54-60. 